\title{
(6) OPEN ACCESS \\ Increasing incidence of non-valvular atrial fibrillation in the UK from 2001 to 2013
}

\author{
Carlos Martinez, ${ }^{1}$ Anja Katholing, ${ }^{1}$ Christopher Wallenhorst, ${ }^{1}$ Serena Granziera, ${ }^{2}$ \\ Alexander T Cohen, ${ }^{3}$ Saul Benedict Freedman ${ }^{4}$
}

\begin{abstract}
- Additional material is published online only. To view please visit the journal online (http://dx.doi.org/10.1136/ heartjnl-2015-307808).

${ }^{1}$ Institute for Epidemiology, Statistics and Informatics GmbH, Frankfurt, Germany ${ }^{2}$ Department of Medicine, University hospital of Padua, Padua, Italy

${ }^{3}$ Department of Haematology, Guy's and St Thomas' Hospitals, King's College, London, UK

${ }^{4}$ Department of Cardiology, Sydney Medical School, University of Sydney, Dept of Cardiology Concord Hospital and Anzac Research Institute, Sydney, Australia
\end{abstract}

\section{Correspondence to} Dr Carlos Martinez, Institute for Epidemiology, Statistics and Informatics $\mathrm{GmbH}$, Im Dinkelfeld 32, 60388 Frankfurt, Germany; carlos martinez@pharmaepi.com

Received 12 March 2015 Revised 26 June 2015 Accepted 3 August 2015 Published Online First 25 August 2015

\section{CrossMark}

\author{
To cite: Martinez $C$, \\ Katholing A, Wallenhorst C, \\ et al. Heart
}

2015;101:1748-1754.

\section{ABSTRACT \\ Objective To determine whether the incidence of atrial fibrillation (AF) is static or rising in the UK.}

Design Among the cohort of all individuals aged $\geq 45$ years in the UK Clinical Practice Research Datalink (CPRD) (linked to hospital discharges) we identified incident non-valvular AF cases between 2001 and 2013. Overall and annual AF incidence rates were calculated and standardised to the UK population.

Results The cohort of 2.23 million individuals included 91707 patients with incident AF. The overall standardised AF incidence rate was $6.7(95 \% \mathrm{Cl} 6.7$ to 6.8) per 1000 person-years, increasing exponentially with age and higher in men of all ages. There was a small increase in the standardised incidence of $A F$ in the last decade from 5.9 (5.8 to 6.1$) / 1000$ person-years in 2001 to 6.9 (6.8 to 7.1)/1000 person-years in 2013, mostly attributable to subjects aged $>80$ years with a non-primary hospital discharge diagnosis of AF.

Standardised incidence rates of $\mathrm{AF}$ among white patients was 8.1 (8.1 to 8.2)/1000 person-years, compared with 5.4 (4.6 to 6.3) for Asians and 4.6 (4.0 to 5.3) for black patients. AF diagnosis was first made in general practice in $39 \%$ of incident AF.

Conclusions The incidence of AF in the UK has increased gradually in the last decade, with more than 200000 first-ever non-valvular AF cases expected in 2015. This increase is only partly due to population ageing, though the principal increase has been in the elderly hospitalised for a reason other than AF.

\section{INTRODUCTION}

Atrial fibrillation (AF) is the most prevalent serious cardiac arrhythmia and a leading cause of secondary thromboembolism. ${ }^{1}$ The ischaemic stroke rate among patients with non-valvular AF averages 5\% per year, ${ }^{2-5}$ two to seven times the rate of those in sinus rhythm depending on the risk score, and mortality is increased twofold. ${ }^{6} 7$

In Western countries, population studies have reported overall incidence rates of AF between 1.9 and 9.9 per 1000 person-years. $^{3}{ }^{8-12}$ Substantial epidemiological uncertainties remain as to why $\mathrm{AF}$ incidence rates differ so widely between studies. In part, this may stem from a variety of methodological differences such as including valvular AF, the method of case definition, study setting, study period, ethnicity and age restriction. ${ }^{12}$ Reliance on data derived only from hospital discharge in some studies $^{5}$ does not account for the considerable numbers diagnosed only in primary care. Few of the published studies report age-specific and gender-specific AF incidence rates making it difficult to determine reasons for the differences.

Age-adjusted incidence rates of AF have increased worldwide, in men by approximately $28 \%$ and in women by $36 \%$ from 1990 to $2010 .^{13}$ This rise is multifactorial and explained only in part by the ageing of the population. ${ }^{12}$ Other contributing factors discussed are secular trends of the increasing prevalence of predisposing factors, that is, systemic hypertension, diabetes mellitus, heart failure, myocardial infarction, valvular heart disease and obesity, which increase arterial stiffness and cause diastolic dysfunction and atrial volume overload. $^{13}$

We conducted a large population-based study to provide contemporary estimates of the incidence rate of $\mathrm{AF}$, and time trends of $\mathrm{AF}$ incidence, and to determine the practice setting of presentation of patients with incident AF.

\section{METHODS}

\section{Data source}

This study used data from the subset of individuals in the UK Clinical Practice Research Datalink (CPRD) who were linked to the Hospital Episodes Statistics (HES). ${ }^{14}$ CPRD is based on primary care and includes demographics, medical history, symptoms and diagnoses recorded with Read medical codes, unstructured medical notes and prescriptions issued by the general practitioner (GP). HES include ethnic origin, dates of hospital admission and discharge, primary and other main reasons for treatment recorded with International Classification of Diseases, Tenth Revision, and surgical operations and procedures performed during hospital stay recorded with the Classification of Interventions and Procedures V.4 of the Office of Population Censuses and Surveys.

\section{Study design}

Generation of study cohort

We conducted a population-based cohort study. The cohort consisted of individuals in the CPRD population aged 45 years and older who were eligible for linkage with additional HES data between 1 January 2001 and 31 December 2013. Patients were included in the cohort from 1 January 2001, provided they had completed at least 1 year of activity in the CPRD. Subjects with a diagnosis of AF prior to cohort entry were excluded. Patients were followed over time until the earliest of 31 December 2013, the first diagnosis of AF, the 
patient died or transferred out of practice, or the end of data collection of the GP practice.

\section{Generation of incident AF cases}

Incident AF cases were identified from primary care-managed episodes and hospital episodes using medical codes from CPRD and HES, respectively. According to the source of the first recording, $\mathrm{AF}$ cases were allocated to either primary care or hospital-based (primary or non-primary discharge diagnosis). To restrict cases to the first occurrence of AF, patients were required to have no previous recording of use of oral anticoagulants (ACs), parenteral therapeutic AC, quinidine, sotalol, amiodarone, flecainide or propafenone, and no prior recording of irregular heartbeats or of a cardioversion (figure 1). Cases were also required to have no previous diagnosis of mitral valve disease (mitral regurgitation, mitral stenosis or mixed mitral valve disease) nor a history of valvular heart surgery. The event date of first AF defined the subject's index day. The index day was shifted to the start of AC treatment only if AC use preceded the first $\mathrm{AF}$ diagnosis by up to 14 days.

The GP's role includes opportunistic pulse checks to detect irregular rhythms that might be AF, in particular in those over 65 years. ${ }^{15}$ The pulse would also be checked during a consultation triggered by symptoms potentially indicative of AF, with an ECG performed in those with an irregular pulse. In our previous study, ${ }^{16}$ we found that just over half of $\mathrm{AF}$ incidence in primary care was detected incidentally in the absence of symptoms.

\section{Data analysis}

Descriptive statistics were used to present the demographic characteristics of incident AF cases. Differences between any of the three groups: (primary care AF, hospital AF with primary discharge diagnosis and hospital AF with non-primary discharge diagnosis) were tested using an F-Test for comparison of means, and Fisher's exact test for comparison of proportions. Crude incidence $\mathrm{AF}$ rates were calculated from the number of new $\mathrm{AF}$

\begin{tabular}{|c|}
\hline $\mathbf{6 , 1 7 9 , 5 1 1} \begin{array}{l}\text { Subjects in data source fulfilling quality criteria } \\
\text { and active any time during study period }\end{array}$ \\
$\qquad$\begin{tabular}{|}
$3,947,669$ Excluded \\
$3,731,161$ Age $<45$ years at end of study period \\
$172,907 \leq 365$ days of observation \\
43,601 AF recorded before $01 / 01 / 2001$
\end{tabular} \\
\cline { 2 - 3 }
\end{tabular}

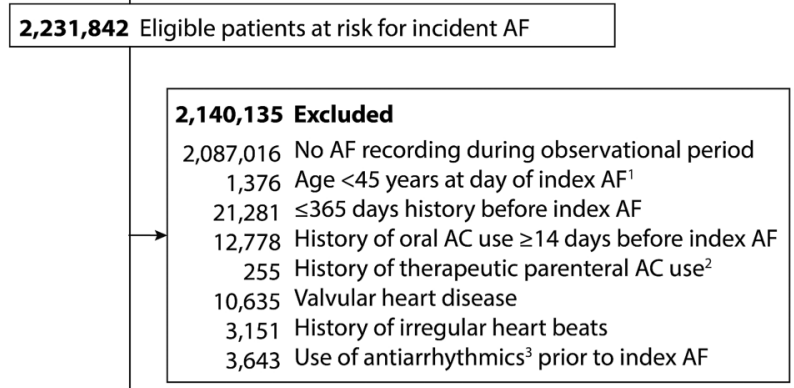

91,707 Incident oral AC-naive non-valvular AF cohort

AF: Atrial fibrillation; AC: anticoagulant

' AF day defined as day of general practioner recording of AF or hospital admission day.

2 Prescription for a parenteral AC in therapeutic dose during the year before index AF day.

${ }_{3}^{3}$ Ouinidine, sotalol, amiodarone, flecainide or propafenone.

Figure 1 Ascertainment of incident atrial fibrillation (AF) cohort (2001-2013). cases and divided by the sum of all individual person-years of the study cohort during the study period. Crude incidence rates were stratified by age, gender, source of $\mathrm{AF}$ diagnosis and calendar year of AF event. Annual overall and ethnic-specific incidence rates of $\mathrm{AF}$ diagnosis standardised using the direct method, applying age-specific and gender-specific crude incidence rates to the UK population. Ethnic-specific incidence rates of AF were estimated for the subset of cohort members with at least one recorded hospitalisation for any cause in HES.

The estimated annual number of new AF cases in 2015 was calculated from age-specific and gender-specific incidence rates of $\mathrm{AF}$ in the years 2011-2013 and the respective projection of the age and gender distribution of the UK population for $2015 .^{17}$

A variance-weighted linear regression model was used to investigate the change of the incidence rates of $\mathrm{AF}$ over time (2001-2013) in men and women separately. In addition, the difference between men and women in change of the incidence rates was estimated by including an interaction term for calendar year and gender in the model with all cases. Two-sided Wald tests were used to test the statistical significance of the genderspecific changes in incidence rates over time and of the interaction between gender and calendar year. All statistical procedures were performed using the STATA MP V.13.1 (StataCorp LP). The Independent Scientific Advisory Committee for CPRD research approved the study protocol (11_024RA).

\section{RESULTS}

The study cohort consisted of 2.23 million subjects with a total of 14.7 million person-years at risk for AF from 2001 to 2013. Within this cohort we identified a total of 91707 incident cases of non-valvular AF (figure 1). Of the total AF cases, 35788 (39.0\%) were recorded in primary care only (largely ambulatory AF) while the remainder 55919 (61.0\%) had an index diagnosis in a hospital: $13474(24.1 \%)$ with a primary discharge diagnosis of AF, and 42445 (75.9\%) with a non-primary AF discharge diagnosis. The mean age of patients with a primary care diagnosis was 74.9 years compared with 73.2 years in those with a primary discharge diagnosis and 78.8 years in those with a nonprimary discharge diagnosis.

The most frequent source of AF diagnosis among those aged 45-79 years was primary care (44\%), and did not differ in the older age groups ( $43 \%$ for those aged $75-79$ years). In contrast, there were marked age differences in those with hospital AF diagnoses, directionally different for primary and non-primary AF discharge diagnoses. Primary AF discharge diagnoses accounted for $26 \%$ of AF detected in those aged 45-59 years, and only $14 \%$ of those aged $75-79$ years, while a non-primary hospital discharge diagnosis accounted for $30.7 \%$ of $\mathrm{AF}$ detected in those aged $45-59$ years, and $43.2 \%$ in those aged 75-79 years. More than half (55.7\%) of those aged 80 years and older had their first AF diagnoses as a non-primary discharge diagnosis. The proportion of women was slightly lower in those with a primary care AF diagnosis (47.0\%), than in those with a primary discharge AF diagnosis (51.9\%) or a nonprimary discharge diagnosis $(51.8 \%)$.

The mean body mass index (BMI) was 27.3 , with $17.4 \%$ having a BMI of 30-35 (stage 1 obesity), and $9.2 \%$ a BMI $\geq 35$ (stage 2 obesity). The proportion underweight was approximately twofold higher in patients with a non-primary discharge diagnosis of $\mathrm{AF}$ than in those with a primary discharge diagnosis of $\mathrm{AF}$ or those found in primary care, while the proportion with stage 1 obesity or stage 2 obesity was lower than in the other two groups (table 1). The majority of the cohort had either never smoked (51.0\%) or was not smoking at the time of 
the diagnosis (36.2\%), while only $12.8 \%$ were current smokers. The prevalence of AF in the first (least deprived), second and third quintiles of the socioeconomic status was higher than in the fourth and fifth quintiles (table 1).

Patients with a non-primary hospital AF discharge diagnosis had a higher prevalence of comorbid conditions than those with a primary discharge diagnosis or with primary care AF. These comorbidities included heart failure, diabetes, history of stroke/ transient ischaemic attack, thromboembolism, myocardial infarction, peripheral vascular disease, chronic pulmonary disease and cerebrovascular disease (table 1). The five most common primary discharge diagnoses were: cerebral infarction,

Table 1 Baseline characteristics of incident AF cohort

\begin{tabular}{|c|c|c|c|c|c|}
\hline & \multirow[b]{2}{*}{ Primary care AF } & \multicolumn{2}{|l|}{ Hospital AF } & \multirow[b]{2}{*}{ Complete AF cohort } & \multirow[b]{2}{*}{ p Value } \\
\hline & & Primary discharge $\mathrm{dx}$ & Non-primary discharge $\mathrm{dx}$ & & \\
\hline Total & $35788(39.0)$ & $13474(14.7)$ & $42445(46.3)$ & $91707(100.0)$ & \\
\hline Age $($ mean $\pm S D)$, years & $74.9 \pm 10.7$ & $73.2 \pm 12.0$ & $78.8 \pm 10.8$ & $76.4 \pm 11.1$ & $<0.001$ \\
\hline Age $45-49$ & $605(1.7)$ & $464(3.4)$ & $423(1.0)$ & $1492(1.6)$ & \\
\hline Age $50-54$ & $1019(2.8)$ & $614(4.6)$ & $681(1.6)$ & $2314(2.5)$ & \\
\hline Age $55-59$ & $1757(4.9)$ & $982(7.3)$ & $1306(3.1)$ & $4045(4.4)$ & \\
\hline Age 60-64 & $2845(7.9)$ & $1331(9.9)$ & $2330(5.5)$ & $6506(7.1)$ & \\
\hline Age $65-69$ & $4024(11.2)$ & 1561 (11.6) & $3443(8.1)$ & $9028(9.8)$ & \\
\hline Age 70-74 & $5536(15.5)$ & $1761(13.1)$ & $5020(11.8)$ & $12317(13.4)$ & \\
\hline Age $75-79$ & $6663(18.6)$ & $2177(16.2)$ & $6723(15.8)$ & $15563(17.0)$ & \\
\hline Age $80-84$ & $6538(18.3)$ & $2071(15.4)$ & 8368 (19.7) & $16977(18.5)$ & \\
\hline Age $85-89$ & $4457(12.5)$ & $1511(11.2)$ & $7731(18.2)$ & $13699(14.9)$ & \\
\hline Age $90+$ & $2344(6.5)$ & $1002(7.4)$ & $6420(15.1)$ & 9766 (10.6) & \\
\hline Female gender & $16838(47.0)$ & 6991 (51.9) & $21968(51.8)$ & $45797(49.9)$ & $<0.001$ \\
\hline Body mass index $(m e a n \pm S D)$ & $27.8 \pm 5.7$ & $27.8 \pm 6.0$ & $26.8 \pm 5.8$ & $27.3 \pm 5.8$ & $<0.001$ \\
\hline$<18.5$ (underweight) & $582(1.8)$ & $263(2.2)$ & $1484(4.2)$ & $2329(2.9)$ & \\
\hline 18.5 to $<25$ (normal weight) & $9768(30.9)$ & 3740 (31.9) & $12840(36.0)$ & $26348(33.4)$ & \\
\hline 25 to $<30$ (overweight) & $12162(38.5)$ & $4434(37.8)$ & $12699(35.6)$ & $29295(37.1)$ & \\
\hline 30 to $<35$ (stage 1 obesity) & $5942(18.8)$ & 2064 (17.6) & $5771(16.2)$ & $13777(17.4)$ & \\
\hline $35+$ (stage 2 obesity) & $3129(9.9)$ & $1236(10.5)$ & $2874(8.1)$ & $7239(9.2)$ & \\
\hline Unknown & $4205(11.7)$ & $1737(12.9)$ & $6777(16.0)$ & $12719(13.9)$ & \\
\hline \multicolumn{6}{|l|}{ Smoking status } \\
\hline Never & $18295(53.2)$ & $6685(52.0)$ & $19729(48.9)$ & $44709(51.0)$ & $<0.001$ \\
\hline Ex & 12520 (36.4) & 4443 (34.5) & 14736 (36.5) & 31699 (36.2) & $<0.001$ \\
\hline Current & 3559 (10.4) & 1735 (13.5) & $5911(14.6)$ & $11205(12.8)$ & $<0.001$ \\
\hline Unknown & $1414(4.0)$ & $611(4.5)$ & $2069(4.9)$ & $4094(4.5)$ & $<0.001$ \\
\hline \multicolumn{6}{|l|}{ Socioeconomic status (SES) } \\
\hline 1st quintile (least deprived) & $8477(24.3)$ & $2948(22.4)$ & 8209 (19.7) & $19634(21.8)$ & $<0.001$ \\
\hline 2nd quintile & $9103(26.0)$ & $3167(24.1)$ & $9786(23.4)$ & $22056(24.5)$ & $<0.001$ \\
\hline 3rd quintile & 7494 (21.4) & $2661(20.2)$ & $8728(20.9)$ & $18883(21.0)$ & 0.014 \\
\hline 4th quintile & $5873(16.8)$ & 2428 (18.4) & $8043(19.3)$ & $16344(18.2)$ & $<0.001$ \\
\hline 5th quintile (most deprived) & $4007(11.5)$ & $1962(14.9)$ & $6979(16.7)$ & $12948(14.4)$ & $<0.001$ \\
\hline Unknown SES & $834(2.3)$ & $308(2.3)$ & $700(1.6)$ & $1842(2.0)$ & $<0.001$ \\
\hline \multicolumn{6}{|l|}{ Ethnic origin $\ddagger$} \\
\hline Asian & $75(0.2)$ & $32(0.2)$ & $84(0.2)$ & $191(0.2)$ & 0.661 \\
\hline Black & $93(0.3)$ & $54(0.4)$ & $144(0.3)$ & $291(0.3)$ & 0.024 \\
\hline Mixed & $27(0.1)$ & $11(0.1)$ & $38(0.1)$ & $76(0.1)$ & 0.795 \\
\hline South Asian & $142(0.4)$ & $79(0.6)$ & $242(0.6)$ & $463(0.5)$ & $<0.001$ \\
\hline White & $28405(85.0)$ & $11709(86.9)$ & $36447(85.9)$ & $76561(85.7)$ & $<0.001$ \\
\hline Other & $147(0.4)$ & $62(0.5)$ & $214(0.5)$ & $423(0.5)$ & 0.157 \\
\hline Unknown & $4528(13.5)$ & $1527(11.3)$ & $5276(12.4)$ & $11331(12.7)$ & $<0.001$ \\
\hline \multicolumn{6}{|l|}{ Comorbidities } \\
\hline Hypercholesterolaemia & $11907(33.3)$ & 4458 (33.1) & $13510(31.8)$ & $29875(32.6)$ & $<0.001$ \\
\hline Lipid lowering drugs & $12733(35.6)$ & $4943(36.7)$ & $16583(39.1)$ & $34259(37.4)$ & $<0.001$ \\
\hline Heart failure & $2894(8.1)$ & $1093(8.1)$ & 5659 (13.3) & $9646(10.5)$ & $<0.001$ \\
\hline Hypertension & $20609(57.6)$ & $7395(54.9)$ & $25496(60.1)$ & $53500(58.3)$ & $<0.001$ \\
\hline Diabetes & $5119(14.3)$ & $2010(14.9)$ & $7857(18.5)$ & $14986(16.3)$ & $<0.001$ \\
\hline Stroke/TIA & $4209(11.8)$ & $1396(10.4)$ & $6517(15.4)$ & $12122(13.2)$ & $<0.001$ \\
\hline Thromboembolism & $403(1.1)$ & $139(1.0)$ & $685(1.6)$ & $1227(1.3)$ & $<0.001$ \\
\hline Myocardial Infarction & $2717(7.6)$ & $1235(9.2)$ & $5133(12.1)$ & $9085(9.9)$ & $<0.001$ \\
\hline Coronary arterial disease without myocardial infarction & $4279(12.0)$ & $1824(13.5)$ & $6646(15.7)$ & $12749(13.9)$ & $<0.001$ \\
\hline Peripheral vascular disease & $3018(8.4)$ & $1152(8.5)$ & 5030 (11.9) & $9200(10.0)$ & $<0.001$ \\
\hline
\end{tabular}


Table 1 Continued

\begin{tabular}{|c|c|c|c|c|c|}
\hline & \multirow[b]{2}{*}{ Primary care AF } & \multicolumn{2}{|l|}{ Hospital AF } & \multirow[b]{2}{*}{ Complete AF cohort } & \multirow[b]{2}{*}{ p Value } \\
\hline & & Primary discharge $\mathrm{dx}$ & Non-primary discharge $\mathrm{dx}$ & & \\
\hline Coronary artery bypass graft & $374(1.0)$ & $158(1.2)$ & $405(1.0)$ & $937(1.0)$ & 0.076 \\
\hline Stent & $610(1.7)$ & $315(2.3)$ & $927(2.2)$ & $1852(2.0)$ & $<0.001$ \\
\hline Chronic pulmonary disease & $7324(20.5)$ & $3117(23.1)$ & $11136(26.2)$ & $21577(23.5)$ & $<0.001$ \\
\hline Cerebrovascular disease & $3847(10.7)$ & $1335(9.9)$ & $6500(15.3)$ & $11682(12.7)$ & $<0.001$ \\
\hline Dementia & $672(1.9)$ & $309(2.3)$ & $2369(5.6)$ & $3350(3.7)$ & $<0.001$ \\
\hline Connective tissue disease & $2055(5.7)$ & $850(6.3)$ & $3219(7.6)$ & $6124(6.7)$ & $<0.001$ \\
\hline Charlson index & $1.4 \pm 1.6$ & $1.5 \pm 1.7$ & $2.1 \pm 2.0$ & $1.7 \pm 1.9$ & $<0.001$ \\
\hline
\end{tabular}

pneumonia, heart failure, acute myocardial infarction and chronic obstructive pulmonary disease while the five most common surgical interventions were coronary artery bypass graft, closed reduction of fracture of bone and internal fixation, prosthetic replacement of head of femur using cement, prosthesis of lens and excision of rectum.

With respect to stroke risk score at baseline, patients with a primary care AF diagnosis had higher mean $\mathrm{CHADS}_{2}$ and $\mathrm{CHA}_{2} \mathrm{DS}_{2}$ VASc scores (1.6 and 3.2, respectively) compared with those with a hospital primary AF discharge diagnosis 1.5 and 3.0), but significantly lower than those with a non-primary discharge diagnosis of AF (1.9 and 3.7), (not shown).

\section{Incidence}

The overall crude incidence rate of AF in those 45 years and older was 6.2 (95\% CI 6.2 to 6.3) per 1000 person-years, 6.6 (6.5 to 6.6) for men and 5.9 (5.9 to 6.0) for women (online supplementary appendix table 2). The overall standardised incidence rate was 6.7 (6.7 to 6.8) per 1000 person-years (online supplementary appendix table 1$)$.

There was a strong and exponential association of AF with increasing age. Incident $\mathrm{AF}$ was diagnosed in 0.6 (0.5 to 0.6) per 1000 person-years at age $45-49$ years, 4.3 (4.2 to 4.3$)$ at age 60-69 years, $11.6(11.4$ to 11.7$)$ at age $70-79$ years, 25.1 (24.8 to 25.4 ) at age $80-89$ years and 40.6 (39.8 to 41.4 ) in those $\geq 90$ years. This age relationship was most marked for non-primary hospital AF diagnoses (figure 2, right panel). AF was more frequent in men of all ages (figure 2, left panel, and online supplementary appendix table 1).

The breakdown by ethnic origin showed a higher standardised incidence rate of AF among white patients of 8.1 (95\% CI 8.1 to 8.2$)$ per 1000 person-years, compared with 5.4 (4.6 to 6.3) for Asians and 4.6 (4.0 to 5.3) for black patients (table 2).

\section{Time trends}

The overall crude incidence rate of AF increased from 5.6 (5.5 to 5.8$)$ per 1000 person-years in 2001 to 6.4 (6.2 to 6.5$)$ in 2013. In men the increase was from $5.8(5.6$ to 6.0$)$ to $7.1(6.8$ to 7.3) per 1000 person-years (plus 22\%, p<0.0001 for the time trend of the incidence rate), and in women it was 5.5 (5.3 to 5.7 ) to 5.8 (5.6 to 6.0 ) per 1000 person-years (plus 5\%, $\mathrm{p}=0.022$ ). The interaction between gender and calendar year was statistically significant $(p=0.0028)$ indicating a difference between the two genders in the increase of the incidence rate of $\mathrm{AF}$ over time. The overall crude incidence rate of first AF when standardised to the age and gender distribution of the UK population over 45 years increased from 5.9 (5.8 to 6.1) per 1000 person-years in 2001 to 6.9 (6.8 to 7.1 ) per 1000 personyears in 2013 (figure 3 and online supplementary appendix table 2). This rise over the last 13 years is paralleled by an increasing AF incidence in those aged $\geq 80$ years (figure 4 , left panel) particularly with a non-primary AF discharge diagnosis, that is, hospital stay unrelated to AF (figure 4, right panel). The annual number of new cases of non-valvular AF expected to occur in 2015 in the UK is 203000,104000 in men and 99000 in women.

\section{DISCUSSION}

We have described the current main epidemiological features of a very large cohort with incident non-valvular AF in the UK, and the temporal trends of standardised AF incidence over the last 13 years. The most striking finding was that the actual standardised AF incidence increased by $16.9 \%$, and that the major contribution to this increased incidence was carried in the very elderly (age $\geq 80$ years), and more particularly in the very elderly hospitalised patients with a non-primary AF discharge diagnosis. This fits with clinical impressions of increased numbers of elderly patients admitted to hospital for either a medical condition or surgery who either are first diagnosed or develop AF during their hospital stay. ${ }^{18}$ Additionally, we described the characteristics of patients depending on the practice setting of their AF diagnosis. Those with a secondary hospital AF diagnosis were older than those with either a primary hospital AF diagnosis or a primary care AF diagnosis.

The important feature of our study is that data are derived from a source population of 6.1 million patients, and include primary care and hospital discharge information derived from very recent data. This point is crucial as over $40 \%$ of incident $\mathrm{AF}$ diagnoses in those aged over 60 years came from primary care, in contrast with many previous studies based solely on hospital discharge or solely on primary care diagnoses. ${ }^{2} 519$ Our population with $\mathrm{AF}$ is therefore more inclusive and likely to represent the characteristics of the entire population, including a broader spectrum of minimal-risk to high-risk patients, as well as a broader age spectrum, given the very different mean ages of patients according to presentation site. ${ }^{6}$

\section{The incidence rate of $\mathrm{AF}$}

The incidence rate of AF increased strongly with age independent of gender and source of presentation as noted in many previous studies. Our overall incident rates were higher than in previous UK studies, ${ }^{2} 19$ two large (German and North 

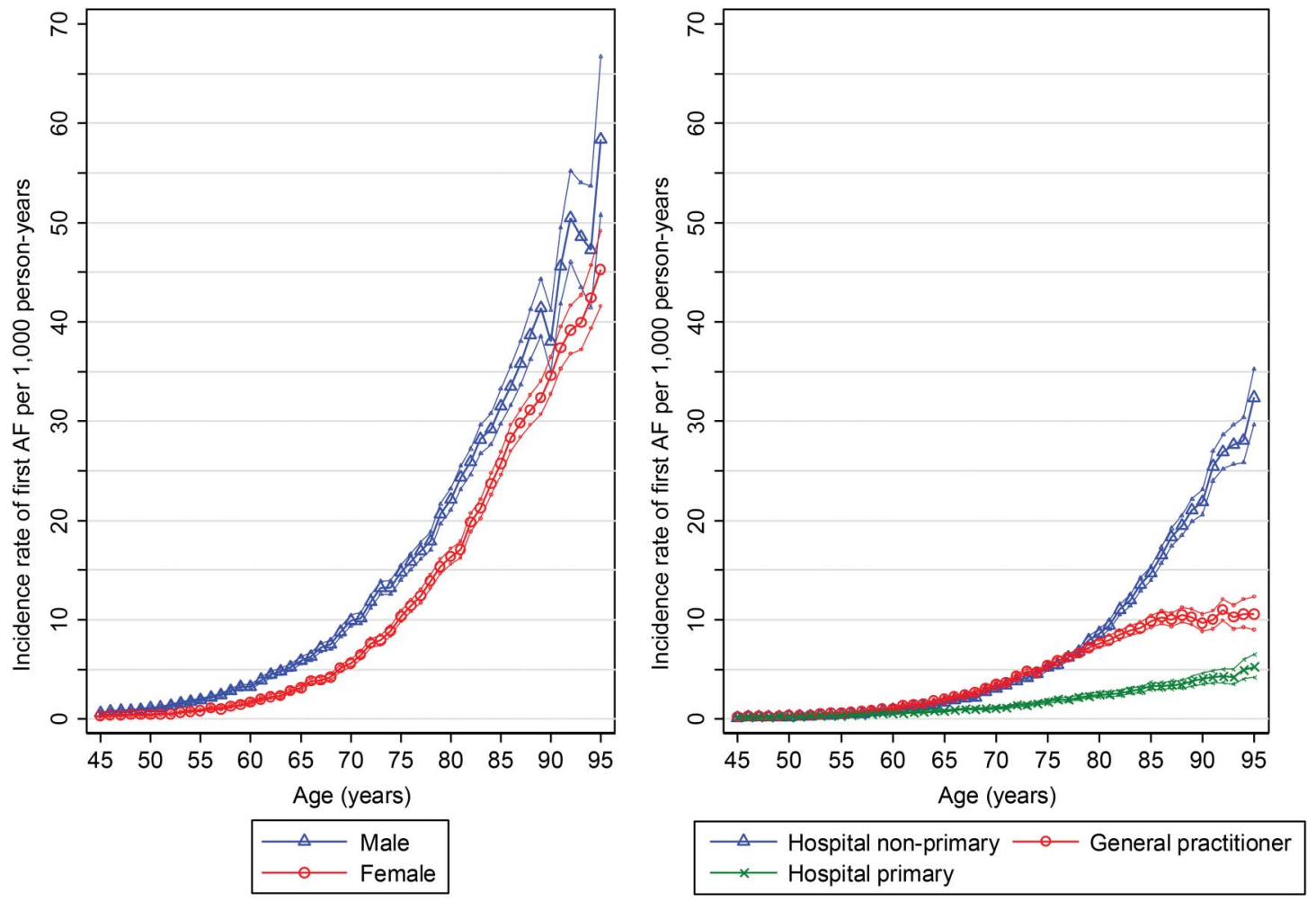

Figure 2 Crude incidence rates of atrial fibrillation (AF) per 1000 person-years with $95 \% \mathrm{Cls}$.

American) cohorts ${ }^{4}{ }^{10}$ and other smaller cohorts. ${ }^{1} 1120$ This may be due to different age restrictions of the sample populations $^{1} 344_{10} 1120$ as there was no upper age restriction in our study and the different sources for identifying AF, as we identified 39\% of all AF in primary care. Additional differences include the mode of AF diagnosis assessment ${ }^{11120}$ as we used coding rather than ECG assessment, and incident AF case definition $^{1420}$ and excluded any condition or medication potentially associated with a prevalent $\mathrm{AF}$ diagnosis. Furthermore, we excluded all AF cases potentially linked with valvular disease. Our age-specific incidence rates were significantly higher than incident rates found in two other cohorts in the UK, ${ }^{25}$ neither of which included hospital discharge, and similar to those in Canada, ${ }^{3}$ and slightly lower than in a prospective cohort study

Table 2 AF incidence by ethnic origin (45 years and older)

\begin{tabular}{lrlll}
\hline $\begin{array}{l}\text { Ethnic } \\
\text { origin }\end{array}$ & Cases & $\begin{array}{l}\text { PT } \\
\text { (years) }\end{array}$ & $\begin{array}{l}\text { Crude incidence rate } \\
\text { (1/1000 years) }\end{array}$ & $\begin{array}{l}\text { Standardised } \\
\text { incidence rate } \\
\text { (1/1000 years) }\end{array}$ \\
\hline Asian & 191 & 49555 & $3.9(3.3$ to 4.4$)$ & $5.4(4.6$ to 6.3$)$ \\
Black & 291 & 93987 & $3.1(2.8$ to 3.5$)$ & $4.6(4.0$ to 5.3$)$ \\
Mixed & 76 & 19090 & $4.0(3.1$ to 5.0$)$ & $5.7(4.3$ to 7.0$)$ \\
South Asian & 463 & 126584 & $3.7(3.3$ to 4.0$)$ & $4.7(4.3$ to 5.2$)$ \\
White & 76561 & 8598166 & $8.9(8.8$ to 9.0$)$ & $8.1(8.1$ to 8.2$)$ \\
Other & 423 & 88961 & $4.8(4.3$ to 5.2$)$ & $6.1(5.5$ to 6.7$)$ \\
Unknown & 11331 & 1959495 & $5.8(5.7$ to 5.9$)$ & $6.3(6.1$ to 6.4$)$ \\
Total & 89336 & 10935838 & $8.2(8.1$ to 8.2$)$ & $7.8(7.7$ to 7.8$)$ \\
\hline
\end{tabular}

*Standardised to age and gender distribution of the UK population. AF, atrial fibrillation; PT, person time.

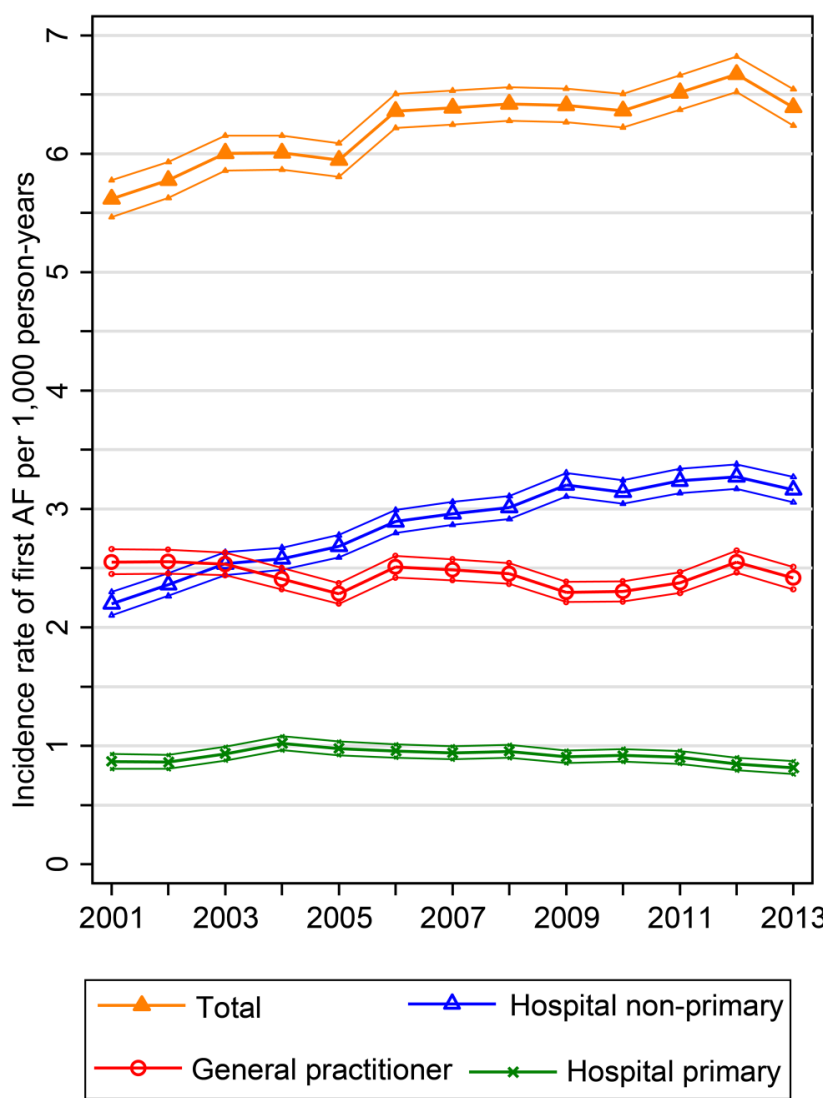

Figure 3 Time trends of crude incidence rates of new atrial fibrillation (AF) per 1000 person-years with $95 \%$ Cls (2001 to 2013) by source of first AF diagnosis. 


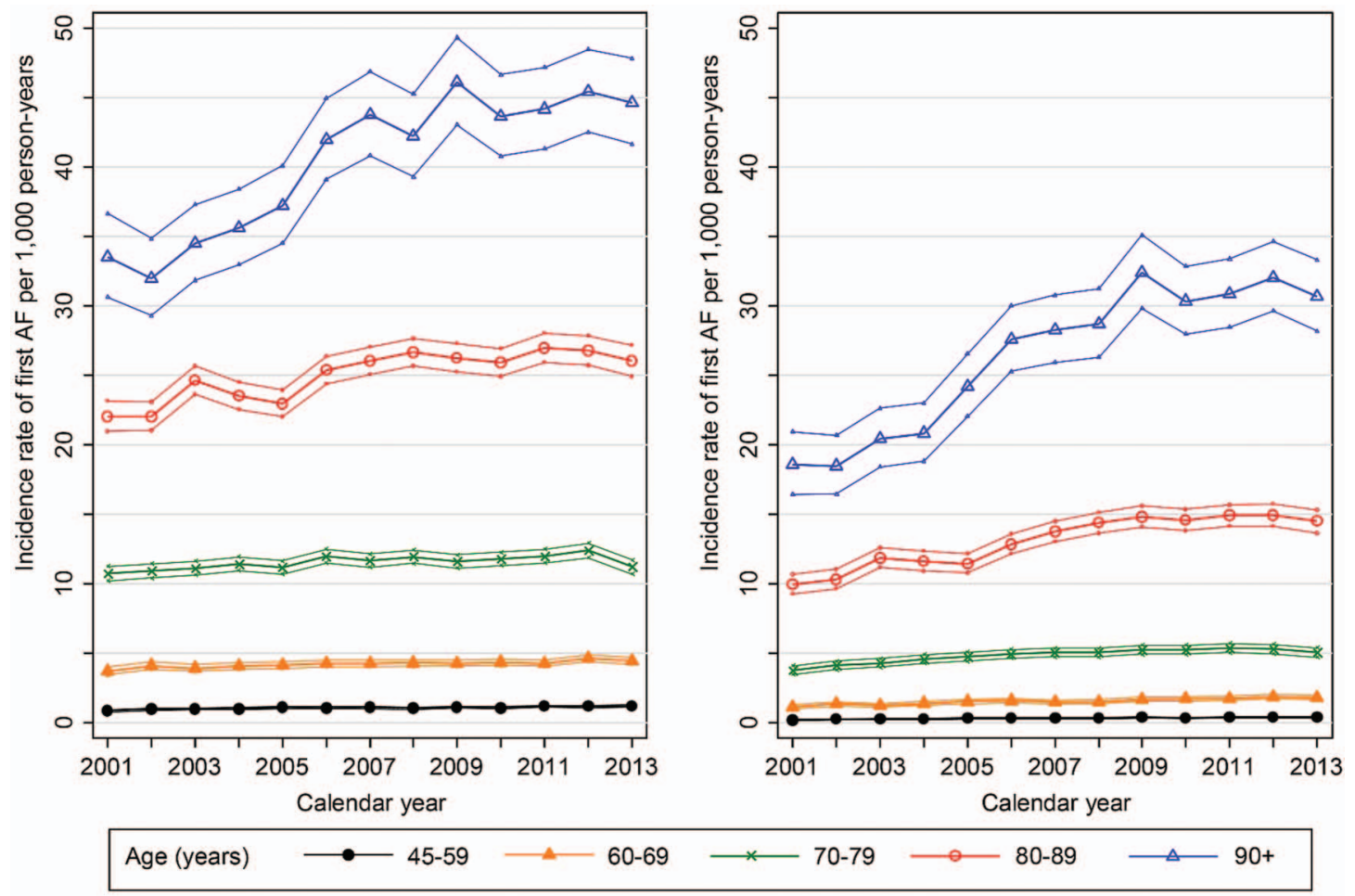

Incidence rates of total AF by age of first AF diagnosis (left panel)

Incidence rates of non-primary hospital discharge diagnosis for AF by age of first AF (right panel)

Figure 4 Time trends of crude incidence rates of atrial fibrillation (AF) per 1000 person-years with 95\% Cls (2001 to 2013 ).

with ECG-confirmed first AF. ${ }^{12}$ The finding of almost twice the incidence rate in white patients compared with black patients, and higher incidence in men than women is consistent with most other studies.

\section{Time trends of AF}

There was a gradual linear increase of incidence rate from 5.9 to 6.9 new AF per 1000 person-years between 2001 and 2013, mostly in men, and almost entirely paralleled by the increase of non-primary hospital AF diagnoses, while incidence rates in general practice and primary AF hospital admissions were static (figure 2, right panel). The increase was also seen in the age-adjusted figures, consistent with the notion that this was not just related to the ageing of the population, and that increases in factors leading to AF were playing a role.

\section{Strengths and limitations}

Our study is based on a large population-based cohort of patients diagnosed with non-valvular AF in primary and secondary care allowing precise estimates of AF incidence. Moreover, the long study period allowed us to describe the time trends over the last 13 years. An original aspect of the paper is the division into primary care diagnosis and secondary care diagnosis groups resulting in a higher sensitivity to detect AF as shown by higher incidence rates of $\mathrm{AF}$ in the UK than previously reported.

The information in each database has its inherent weaknesses. In our study AF was defined by medical codes recorded in primary care or secondary care and not directly by ECG tracings, so misclassification of AF may have occurred. Under-reporting is also possible, since not all concomitant diseases are translated in the hospital discharge codes. Medical codes did not differentiate between paroxysmal, persistent and permanent $\mathrm{AF}$ as we defined $\mathrm{AF}$ as the first recording of any $\mathrm{AF}$ only. Thus the validity of AF in our study depended on its coding accuracy as the underlying criteria leading to the recording of an AF diagnosis are unknown. It is possible that some patients were not true incident cases although we required at least 1 year of information available before cohort entry to ensure sufficient baseline information to exclude prevalent cases, and we also required cohort members to be oral anticoagulant-naïve, to have no history of use of antiarrhythmics and no history of recorded irregular beats. Silent and undetected AF was obviously not recorded.

\section{CONCLUSIONS}

Non-valvular AF is a common medical condition with approximately 200000 new cases annually in the UK. The incident rate increases with age and is higher in men. Many cases are first recorded in primary care, and this large source of diagnosis has been excluded from many previous studies. The incidence rate is gradually rising over the last 13 years, and this results from ageing of the population and from increases in conditions that may predispose to AF. The main contributor to the increasing incidence of $\mathrm{AF}$ is the increasing numbers of elderly people with a secondary diagnosis of AF at hospital discharge. Greater awareness of the increasing incidence of AF in the elderly may lead to increased monitoring for new onset AF in elderly patients in primary care settings and when hospitalised for reasons other than AF. This in turn may facilitate earlier institution of therapy to prevent secondary thromboembolism. 


\section{Key messages}

What is already known on this subject?

Atrial fibrillation (AF) is the most prevalent serious cardiac arrhythmia and a leading cause of secondary thromboembolism. The ischaemic stroke rate among patients with non-valvular AF averages $5 \%$ per year, two to seven times the rate of those in sinus rhythm depending on the risk score, and mortality is increased twofold. Contemporary estimates of the incidence rate and time trends of AF are scarce.

\section{What might this study add?}

Non-valvular AF is common in the UK with approximately 200000 new cases annually. Over the last 13 years the actual standardised AF incidence increased by $17 \%$, and is almost double in white patients than black patients or Asians. The major contribution to this increase is carried in the very elderly (age $\geq 80$ years), particularly those elderly hospitalised patients with a non-primary AF discharge diagnosis.

\section{How might this impact on clinical practice?}

Greater awareness of the increasing incidence of $A F$ in the elderly may lead to increased monitoring for new onset $A F$ in elderly patients in primary care settings and when hospitalised for reasons other than AF. This in turn may facilitate earlier institution of therapy to prevent secondary thromboembolism.

Contributors Conception or design: CM, SBF, ATC. Acquisition and data analysis: $\mathrm{AK}, \mathrm{CW}, \mathrm{CM}$. Interpretation of data and critical revision of the manuscript for important intellectual content: all authors. Drafting of the manuscript: CM, SBF, SG, ATC. Responsibility: CM takes full responsibility for integrity of data and analyses.

Funding This work was in part supported by Bayer Pharma AG, Global Health Economics and Outcomes Research, Wuppertal, Germany. The views expressed are those of the authors and do not necessarily represent the views of the sponsor.

Competing interests CM reports personal fees from Boehringer Ingelheim, grants from CSL Behring, Bayer Pharma AG and BMS-Pfizer, outside the submitted work. AK, CW and SG have nothing to disclose. SBF reports grants, personal fees and non-financial support from Bayer Pharma AG, grants and non-financial support from Boehringer Ingelheim, grants and personal fees from BMS/Pfizer, personal fees from Servier, personal fees from Astra-Zeneca, personal fees from Gilead, outside the submitted work. ATC reports receiving grants and personal fees from Bayer, Boehringer Ingelheim, BMS, Daiichi, Johnson and Johnson, Pfizer, Portola, Sanofi, X01, outside of the submitted work.

Provenance and peer review Not commissioned; externally peer reviewed.

Open Access This is an Open Access article distributed in accordance with the terms of the Creative Commons Attribution (CC BY 4.0) license, which permits others to distribute, remix, adapt and build upon this work, for commercial use, provided the original work is properly cited. See: http://creativecommons.org/ licenses/by/4.0/

\section{REFERENCES}

1 Alonso A, Agarwal SK, Soliman EZ, et al. Incidence of atrial fibrillation in whites and African-Americans: the Atherosclerosis Risk in Communities (ARIC) study. Am Heart J 2009;158:111-17.

2 Rietbrock S, Heeley E, Plumb J, et al. Chronic atrial fibrillation: incidence, prevalence, and prediction of stroke using the Congestive heart failure, Hypertension, Age $>75$, Diabetes mellitus, and prior Stroke or transient ischemic attack (CHADS2) risk stratification scheme. Am Heart J 2008;156:57-64.

3 Renoux C, Patenaude V, Suissa S. Incidence, mortality, and sex differences of nonvalvular atrial fibrillation: a population-based study. J Am Heart Assoc 2014;3:e001402.

4 Piccini JP, Hammill BG, Sinner MF, et al. Incidence and prevalence of atrial fibrillation and associated mortality among Medicare beneficiaries, 1993-2007. Circ Cardiovasc Qual Outcomes 2012;5:85-93.

5 Murphy NF, Simpson CR, Jhund PS, et al. A national survey of the prevalence, incidence, primary care burden and treatment of atrial fibrillation in Scotland. Heart 2007;93:606-12.

6 Camm AJ, Lip GY, De Caterina R, et al. 2012 focused update of the ESC Guidelines for the management of atrial fibrillation: an update of the 2010 ESC Guidelines for the management of atrial fibrillation. Developed with the special contribution of the European Heart Rhythm Association. Eur Heart I 2012;33:2719-47.

7 January CT, Wann LS, Alpert JS, et al. 2014 AHA/ACC/HRS guideline for the management of patients with atrial fibrillation: a report of the American College of Cardiology/American Heart Association Task Force on Practice Guidelines and the Heart Rhythm Society. J Am Coll Cardiol 2014;64:e1-76.

8 Benjamin EJ, Levy D, Vaziri SM, et al. Independent risk factors for atrial fibrillation in a population-based cohort. The Framingham Heart Study. JAMA 1994;271:840-4.

9 Krahn AD, Manfreda J, Tate RB, et al. The natural history of atrial fibrillation: incidence, risk factors, and prognosis in the Manitoba Follow-Up Study. Am J Med 1995;98:476-84.

10 Wilke $\mathrm{T}$, Groth $\mathrm{A}$, Mueller $\mathrm{S}$, et al. Incidence and prevalence of atrial fibrillation: an analysis based on 8.3 million patients. Europace 2013;15:486-93.

11 Heeringa J, van der Kuip DA, Hofman A, et al. Prevalence, incidence and lifetime risk of atrial fibrillation: the Rotterdam study. Eur Heart J 2006;27:949-53.

12 Miyasaka Y, Barnes ME, Gersh BJ, et al. Secular trends in incidence of atrial fibrillation in Olmsted County, Minnesota, 1980 to 2000, and implications on the projections for future prevalence. Circulation 2006;114:119-25.

13 Chugh SS, Havmoeller R, Narayanan K, et al. Worldwide epidemiology of atrial fibrillation: a Global Burden of Disease 2010 Study. Circulation 2014;129:837-47.

14 CPRD link with HES and ONS. Available from http://www.cprd.com/recordLinkage (accessed 24 Feb 2015).

15 Improvement N. Heart and Stroke Improvement. Atrial fibrillation in primary care: making an impact on stroke prevention. National priority project final summaries. 2009. http://www.nhsiq.nhs.uk/media/2335787/af_summaries_2009.pdf (accessed 21 Apr 2015)

16 Martinez C, Katholing A, Freedman SB. Adverse prognosis of incidentally detected ambulatory atrial fibrillation. A cohort study. Thromb Haemost 2014;112: $276-86$.

17 Office for National Statistics. 2010-based National Population Projections. http:// www.ons.gov.uk/ons/publications/re-reference-tables. html? edition=tcm \% 3A77-229866. Published 26 October 2011 (accessed 10 Feb 2015).

18 Hospital Episode Statistics, Admitted Patient Care, England-2013-14. Health and Social Care Information Centre. http://www.hscic.gov.uk/catalogue/PUB16719 (accessed 28 Feb 2015).

19 Ruigomez A, Johansson S, Wallander MA, et al. Incidence of chronic atrial fibrillation in general practice and its treatment pattern. J Clin Epidemiol 2002;55:358-63.

20 Miyasaka Y, Barnes ME, Gersh BJ, et al. Time trends of ischemic stroke incidence and mortality in patients diagnosed with first atrial fibrillation in 1980 to 2000: report of a community-based study. Stroke 2005;36:2362-6. 